\title{
A Versão Brasileira do Three-Dimensional Work Fatigue Inventory (3D-WFI): Adaptação e Propriedades Psicométricas
}

\author{
Alexsandro Luiz De Andrade ${ }^{1}$, Marcos Aguiar de Souza ${ }^{2}$, Pedro Paulo Pires ${ }^{3}$
}

${ }^{1}$ http://orcid.org/0000-0003-4953-0363 / Universidade Federal do Espírito Santo (UFES), Brasil
${ }^{2}$ http://orcid.org/0000-0001-6609-8766 / Universidade Federal do Rio de Janeiro (UFRJ), Brasil
${ }^{3}$ http:// orcid.org/0000-0001-8831-9056 / Universidade Federal do Rio de Janeiro (UFRJ), Brasil

Fadiga no trabalho é a percepção individual de desconforto físico, cognitivo e emocional, associada com sentimentos de perda de energia, cansaço e exaustão, levando a efeitos deletérios no desempenho laboral. O objetivo do presente trabalho foi adaptar e levantar evidências psicométricas para o contexto brasileiro do Three-Dimensional Work. Fatigue Inventory (3D-WFI). O estudo foi conduzido com 455 trabalhadores adultos, oriundos de três contextos ocupacionais (segurança pública, saúde e população geral). Entre estes 251 eram do sexo masculino. Os dados analisados a partir de procedimentos fatoriais confirmatórios e multigrupo apontaram adequação ao modelo original de três dimensões de fadiga (física, cognitiva e emocional). Indicadores de precisão foram bons e superiores a $0,91 \mathrm{em}$ todas as dimensões resultantes. Evidências externas do 3D-WFI foram observadas com engajamento no trabalho, satisfação com a vida e conflito trabalho-família. Discutem-se aplicações do instrumento para o contexto de pesquisa e diagnóstico nas organizações.

Palavras-chave: psicometria, saúde ocupacional, fadiga no trabalho, estresse no trabalho.

\section{The Brazilian Version of the Three-Dimensional Work Fatigue Inventory (3D-WFI): Adaptation and Psychometric Properties}

\section{Abstract}

Work fatigue is the individual's perception of physical, cognitive, and emotional discomfort, associated with feelings of energy loss, tiredness, and exhaustion, leading to deleterious effects on work performance. This study aimed to adapt and raise psychometric evidence for the Brazilian context of the Three-Dimensional Work Fatigue Inventory (3D-WFI). The study was conducted with 455 adult workers from three occupational contexts (public safety, health, and general population). Among these, 251 were male. The data analyzed using confirmatory factorial and multigroup procedures indicated the original threedimensional fatigue model (physical, cognitive and emotional). Accuracy indicators were good and above 0.91 in all resulting dimensions. External evidence of 3D-WFI was observed with work engagement, life satisfaction, and work-family conflict. Applications of the instrument for the context of research and diagnosis in organizations are discussed.

Keywords: psychometry, occupational health, work fatigue, work stress.

\section{La Versión Brasileña del Three-Dimensional Work Fatigue Inventory (3D-WFI): Adaptación y Propiedades Psicométricas}

Resumen

La fatiga en el trabajo es la percepción individual de incomodidad física, cognitiva y emocional, asociada con sentimientos en detrimento de energía, cansancio y agotamiento, que conducen a efectos nocivos en el desempeño laboral. El objetivo de este estudio fue adaptar y levantar evidencias psicometricas de validez para el contexto brasileño del Three-Dimensional Work Fatigue Inventory (3D-WFI). El estudio se realizó con 455 trabajadores adultos, oriundos de tres contextos (seguridad pública, salud y población general), entre estos 251 eran hombres. Los datos analizados a partir de análisis factoriales confirmatorios y multigrupo indicaron idoneidad para el modelo original de tres dimensiones de fatiga (física, cognitiva y emocional). Los indicadores de precisión fueron buenos y superiores a 0,91 en todos los factores. Fueron observadas evidencias externas del 3D-WFI con el compromiso laboral, la satisfacción con la vida y el conflicto trabajo-familia. Se discuten las aplicaciones del instrumento para el contexto de la investigación y el diagnóstico organizacional.

Palabras clave: psicometría, salud laboral, fatiga en el trabajo y estrés en el trabajo. 
Fadiga é a percepção individual de desconforto físico e cognitivo, associada com sentimentos de cansaço e exaustão, levando a impactos negativos na capacidade de trabalhar de maneira segura e eficaz (Frone \& Tidwell, 2016). A fadiga pode levar a diversos problemas, como a perda de habilidades motoras e cognitivas, envolvendo a perda de reflexo e o prejuízo na tomada de decisão e julgamento (Chen, Lu, \& Mao, 2019), no contexto específico de aspectos de trabalho fadiga pode associar-se com maior exposição a riscos e acidentes, adoecimento e problemas interpessoais (Frone \& Blais, 2020; Stuetzle, Pavlin, Smith, \& Weston, 2018).

O construto fadiga no trabalho é objeto de uma recente roupagem teórica e metodológica (Frone \& Blais, 2020; Frone, Reis, \& Ottenstein, 2018; Frone \& Tidwell, 2016). A perspectiva atual de concepção do construto parte da interconexão entre aspectos pessoais (ex. recursos) e consequentes do trabalho (ex. demandas) na manifestação da fadiga, estabelecendo uma relação teórica com a perspectiva de conservação de recursos (Hobfoll, 1989), as demandas do ambiente de trabalho e repercussões para domínios de saúde e vida familiar do indivíduo (Fan \& Smith, 2019; Frone \& Tidwell, 2016; Westman, Hobfoll, Chen, Davidson, \& Laski, 2015).

As definições atuais do conceito de fadiga englobam três eixos centrais (Frone \& Tidwell, 2016). O primeiro diz respeito aos aspectos de sintoma, representado por características de extremo cansaço, bem como redução da capacidade funcional do indivíduo. O segundo relaciona-se aos domínios efetivos de manifestação do comportamento individual, no caso os aspectos: físicos, mentais e emocionais. Por fim, o eixo associado aos aspectos da temporalidade, como por exemplo, um dia, uma semana de trabalho ou um mês da experiência percebida.

Alguns contextos profissionais são comumente remetidos como ambientes de maior probabilidade de fadiga para seus profissionais, é o caso do trabalho em serviços hospitalares (Stuetzle et al., 2018), atuação em emergências (Patterson et al., 2018), controle de trafego aéreo (Chen et al., 2019) e serviços de segurança (Knutsen, 2019). Pesquisas também apontam a incidência elevada de fadiga em profissionais de negócios (Fourie \& Keyser, 2018), mineração (Bauerle, Dugdale, \& Poplin, 2018), motoristas de táxi (Husain, Mohamad, \& Idris, 2019) e profissionais ferroviários (Fan \& Smith, 2019).

As pesquisas sobre causas da fadiga possuem origens diversas. Aspectos organizacionais, como a redução de pessoal e de custos (ex. downsizing) são apontados como fontes de maior fadiga para trabalhadores (Frone \& Blais, 2020). Variáveis do contexto laboral, como aspectos ergonômicos do ambiente do trabalho e características da atividade de trabalho também apresentam-se como antecedentes da fadiga (Chen et al., 2019). Numa outra ponta, estudos procuram investigar também os efeitos da fadiga, como por exemplo, o aumento do conflito trabalho-família (Obeid et al., 2019; Powell \& Greenhaus, 2010).

Para avaliar a fadiga no trabalho são desenvolvidos instrumentos psicométricos de medida (Celestino, Bucher-Maluschke, \& Marqueze, 2020; Frone \& Blais, 2020b; Frone \& Tidwell, 2016). No contexto brasileiro, alguns instrumentos possuem tradição de uso em pesquisas, sendo eles: a) Piper Fatigue Scale (Mota, Pimenta, \& Piper, 2009), b) Escala de avaliação da fadiga (De Vries, Michielsen, Van Heck, \& Drent, 2004; Gouveia et al., 2015); c) Chalder Fatigue Questionnaire (Cho et al., 2007) e; d) The Need for Recovery Scale (NFR) (Moriguchi et al., 2012). No que tange aos modelos teóricos sobre fadiga no trabalho, um ponto de discussão entre distintas opções de medidas é o aspecto da natureza uni ou multidimensional do construto (Gouveia et al., 2015), havendo prevalência na literatura internacional para modelos complexos e de distintas dimensões (Frone \& Blais, 2020b; Frone et al., 2018;
Frone \& Tidwell, 2016).

\section{Three-Dimensional Work Fatigue Inventory (3D-WFI)}

O Three-Dimensional Work Fatigue Inventory (3D-WFI) é um instrumento psicométrico desenvolvido e validado inicialmente numa amostra de mais de 2000 trabalhadores assalariados americanos (Frone \& Tidwell, 2016). A construção do inventário seguiu as etapas metodológicas pertinentes ao desenvolvimento de instrumentos psicológicos, englobando: a) revisão teórica do construto e outras medidas, b) construção de itens e instruções de resposta, c) avaliação piloto; d) levantamento de evidências psicométricas internas, a partir de procedimentos de análise fatorial exploratória e confirmatória e; e) levantamento de evidências externas com outros construtos (satisfação com o trabalho, satisfação com colegas de trabalho, saúde física e mental).

A versão final do 3D-WFI contou com 18 itens equitativamente distribuídos entre três dimensões de fadiga: física, cognitiva e emocional. Os itens foram respondidos por escalas de frequência de eventos de cinco intervalos (todos os dias, ao menos uma vez por semana, ao menos uma vez por mês, menos de uma vez por mês e nunca), as quais demarcam a interpretação de escores baixo nos construtos como indicadores de fadiga (ex. todos os dias) e pontuações elevadas como baixos índices de fadiga (ex. nunca). A tabela 1 apresenta as dimensões e definições constitutivas dos construtos mesurado pelo 3D-WFI.

Tabela 1

Dimensões e definições do 3D-WFI

\begin{tabular}{cc}
\hline Construto & Definição \\
\hline $\begin{array}{c}\text { Fadiga } \\
\text { física }\end{array}$ & $\begin{array}{c}\text { Representa cansaço físico extremo e capacidade reduzida de prati- } \\
\text { car atividades físicas experimentadas durante e ao final da jornada } \\
\text { de trabalho. }\end{array}$ \\
$\begin{array}{c}\text { Fadiga } \\
\text { mental }\end{array}$ & $\begin{array}{c}\text { Diz respeito ao cansaço mental extremo e capacidade reduzida de } \\
\text { se engajar em atividades cognitivas experimentadas durante e ao } \\
\text { final do dia de trabalho. }\end{array}$ \\
$\begin{array}{c}\text { Fadiga } \\
\text { emocional }\end{array}$ & $\begin{array}{c}\text { Corresponde ao cansaço emocional extremo e capacidade reduzi- } \\
\text { da de envolver em atividades emocionais que ocorrem durante } \\
\text { e ao final da jornada de trabalho. }\end{array}$ \\
\hline
\end{tabular}

O WFI-3D pode ser considerado um instrumento bastante atual, todavia versões já foram adaptadas em outros países com êxito. É o caso da versão alemã, adaptada com uma população de trabalhadores daquele país (Frone et al., 2018), obtendo evidências positivas de validade de estrutura interna $(\chi 2=356, p<$ 0,001; CFI = 0,96; TLI = 0,96; SRMR =0,03; e RMSEA =0,06) e precisão (coeficientes de Cronbach superiores a 0,93). Existe também relatos da tradução e teste de confiabilidade (coeficientes de Cronbach superiores a 0,67), em uma versão árabe, utilizada com população de trabalhadores libaneses (Obeid et al., 2019) e uma adaptação do instrumento com redução de itens na Noruega (Knutsen, 2019), obtendo adequada evidência de estrutura interna $(\chi 2=1628, p<0,001 ; \mathrm{CFI}=0,92 \mathrm{TLI}=0,91 ; \mathrm{SRMR}=0,05 ; \mathrm{e}$ RMSEA $=0,05)$ e indicadores de precisão superiores a 0,93 .

\section{Objetivo e Hipóteses}

O objetivo central deste trabalho foi adaptar e levantar evidências psicométrica de validade (estrutura interna e convergente) e precisão para o contexto brasileiro do Work Fatigue Inventory (3D-WFI). Considerando a natureza do padrão de resposta do instrumento, pontuações elevadas demarcam baixa fadiga e escores reduzidos estabelecem a existência de elevada de fadiga (Frone \& Tidwell, 2016), são traçadas quatro hipóteses teórico-empíricas que visam compreender a natureza axiológica da fadiga laboral 
avaliada com o 3D-WFI e os construtos conflito trabalho-família (Netemeyer, Boles, \& McMurrian, 1996), satisfação com a vida (Diener, Emmons, Larsem, \& Griffin, 1985; Zanon, Bardagi, Layous, \& Hutz, 2014) e engajamento no trabalho (Vazquez, Ferreira, \& Mendonça, 2019), além de evidências complementares de validade externa do $3 D-W F I$.

São hipóteses do estudo: H1, H2, H3 e H4.

H1. Considerando os bons indicadores obtidos no desenvolvimento do 3D-WFI (Frone \& Tidwell, 2016) e a replicação da estrutura interna de três dimensões de fadiga na adaptação do instrumento no contexto alemão (Frone et al., 2018), libanês (Obeid et al., 2019) e norueguês (Knutsen, 2019), a hipótese um do estudo norteia-se pela obtenção de evidências positivas de validade (estrutura interna, externa), além de precisão do 3D-WFI no contexto brasileiro.

H2. Compreende-se que aspectos do contexto laboral interagem e afetam dimensões da dinâmica familiar, assim como situações da vida pessoal potencialmente podem impactar o domínio do trabalho (Akinbode, Folusho, \& Isrsel, 2018; Obeid et al., 2019), aspectos que são traduzidos na díade do conflito trabalho-família (De Andrade, Moraes, Martins-Silva, \& Queiroz, 2020; Netemeyer et al., 1996), em trabalho interferir na família e família interferir no trabalho, do construto conflito trabalho-família. Espera-se, na hipótese dois, que pessoas com maior fadiga laboral também possuam maiores índices de conflito trabalho-família, evidenciando uma correlação negativa entre as variáveis avaliadas no estudo.

H3. Sendo a satisfação com a vida uma percepção geral do indivíduo sobre a prevalência de aspectos positivos sobre o negativos na vida (Diener et al., 1985; Zanon et al., 2014), bem como o potencial efeito de transbordamento de emoções negativas do trabalho para domínios da vida e vice versa (Grawitch, Maloney, Barber, \& Mooshegian, 2013). Então espera-se, na hipótese três deste estudo, que pessoas com maior fadiga no trabalho tenham menor percepção de satisfação com a vida, expectando assim uma correlação positiva entre os escores da 3D-WFI e a escala de satisfação com a vida neste estudo.

H4. Por fim, entendendo que o engajamento no trabalho representa um estado psicológico positivo de bem-estar, absorção positiva e identificação com o trabalho (Vazquez et al., 2019) e a fadiga no trabalho um aspecto que consome recursos pessoais e consequentemente propiciadora de sintomas de cansaço excessivo e exaustação (Frone \& Tidwell, 2016). A quarta hipótese do estudo espera que quão mais fadigado no trabalho esteja o indivíduo, menor seja seu engajamento laboral, havendo, dessa forma, a expectativa de uma correlação positiva entre a medida de engajamento no trabalho e o inventário de fadiga laboral adaptado no presente estudo.

\section{Método}

\section{Participantes}

O presente estudo contou com uma amostra de 455 participantes adultos, sendo 251 do sexo masculino (55,2\%) e $204 \mathrm{mu}-$ lheres $(44,8 \%)$. A maioria dos participantes no momento da coleta de dados tinha idade de 30 a 39 anos $(n=208 ; 45,7 \%)$, seguido por participantes de 40 a 49 anos $(n=111 ; 24,4 \%)$. Os participantes foram oriundos de três contextos profissionais: segurança pública ( $n=189 ; 41,5 \%)$, envolvendo uma totalidade de policiais militares, contexto hospitalar $(n=160 ; 35,5 \%)$, envolvendo maioria de enfermeiros e técnicos de saúde e; população geral ( $n=106 ; 23,3 \%$ ). Quanto origem demográfica, a maioria da amostra foi do sudeste $(n=213 ; 46,8 \%)$, seguida pela região norte $(n=191 ; 42 \%)$ e sul $(n=39 ; 8,6 \%)$

\section{Instrumentos}

Procedimentos de adaptação transcultural do instrumento. O Three-Dimensional Work Fatigue Inventory (3D-WFI) foi traduzido e adaptado no Brasil seguindo orientações técnicas e metodológicas da área (Borsa, Damásio, \& Bandeira, 2012; International Test Comission, 2017). Como primeiro passo do processo metodológico, foi solicitada autorização junto ao autor principal da versão original do inventário (Frone \& Tidwell, 2016). Sequencialmente dois tradutores independentes realizaram a tradução da versão original do inventário, sendo sequenciado uma versão síntese pelo pesquisador principal deste artigo, a qual foi retraduzida e apresentada a um terceiro especialista com proficiência em inglês e formação em psicologia. Com devidos ajuste, uma versão nomeada de instrumento piloto foi aplicada em cinco participantes com perfil de idade e ocupação da população alvo. Sem necessidades adicionais de ajuste, o instrumento disponibilizado para levantamento de evidências psicométricas adicionais no estudo de campo.

Por decisão de adaptação de um instrumento fiel as características originais e futuras comparações transculturais, manteve-se a chave de resposta de frequência gradual decrescente (todos os dias, ao menos uma vez por semana, ao menos uma vez por mês, menos de uma vez por mês e nunca), fato que impõem o cuidado da interpretação de escores elevados como indicador de baixa fadiga e escores baixos como indicador de alta fadiga.

Instrumentos utilizados. Os participantes da pesquisa responderam um questionário contendo questões para caracterização da amostra, além de quatro instrumentos psicológicos. Cada um deles, encontra-se descrito a seguir:

a) Caracterização sociodemográfica. Foi feita por meio de um questionário desenvolvido para avaliar características demográficas e socioeconômicas dos participantes que pudessem ser relevantes para a análise, como sexo, idade, renda familiar e curso de formação.

b) Escala de conflito trabalho-família. Instrumento composto por 10 itens adaptados ao contexto brasileiro (Aguiar \& Bastos, 2013) que avaliam as duas dimensões do conflito, a interferência do trabalho na família - TIF (ex. "As demandas do meu trabalho interferem na minha vida familiar", $\alpha=0,90)$ e a interferência da família no trabalho - FIT (ex. "As demandas de minha família interferem nas minhas atividades de trabalho", $\alpha=0,86)$. Os itens foram respondidos por escalas do tipo Likert de seis intervalos (1 $=$ Discordo totalmente a $6=$ Concordo totalmente).

c) Escala de Satisfação com a Vida. A medida de cinco itens avalia aspectos gerais da satisfação com a vida (ex: A minha vida está próxima do meu ideal, $\alpha=0,87$ ). Com evidências psicométricas positiva no Brasil (Zanon, Bardagi, Layous \& Hutz, 2014). Os itens foram respondidos por escalas do tipo Likert de sete intervalos $(1=$ Discordo totalmente a 7 = Concordo totalmente $)$.

d) Escala de Engajamento no Trabalho. Instrumento original de dez itens que avaliam aspectos, crenças de vigor e absorção para no trabalho (Siqueira et al., 2014). (ex. Enquanto trabalho eu acredito que fico cheio de energia; $\alpha=0,78)$. A chave de resposta dos itens foi uma escala de cinco intervalos (nunca (1) a sempre (5).

e) Three-Dimensional Work Fatigue Inventory (3D-WFI). Versão traduzida para o português e adaptada no presente estudo do 3D-WFI (Frone \& Tidwell, 2016). 
Inicialmente o estudo foi avaliado e aprovado pelo Comitê de Ética em Pesquisa da Universidade Federal do Espírito Santo, obtendo aprovação e autorização para coleta de dados (CAAE n. 15422119.2.0000.5542). O processo de recrutamento de participantes foi iniciado a partir de contato telefônico com responsáveis legais entre as organizações de origem dos participantes. Reuniões de alinhamento da proposta do estudo e sobre o processo de coleta foram realizadas anterior ao processo de coleta de dados. Após autorização do responsável institucional, os pesquisadores visitavam os locais de trabalho em horários previamente agendados e realizava a coleta de maneira coletiva em ambiente de sala de treinamento ou reuniões. Todos os participantes da presente pesquisa manifestaram concordância e assinaram o termo de consentimento livre e esclarecido, entregue pelos pesquisadores previamente ao preenchimento do questionário. O tempo total de resposta médio foi de 20 minutos.

\section{Procedimentos de Análise dos Dados}

$\mathrm{O}$ processo analítico contou com auxílio dos softwares $\mathrm{R}$ e Mplus. Primeiramente o banco de dados foi preparado, inspecionado e os dados omissos foram tratados. Para o levantamento de evidências internas de validade do inventário de fadiga no trabalho, assumiu-se a natureza ordinal categórica dos dados e executaram-se procedimentos de análise fatorial confirmatória, com método de estimação MLR (Robust ML). Como passo preliminar, os dados foram investigados através do teste de dimensionalidade de Critério de Informação Bayesiano de Schwarz. Foram testados dois modelos, o primeiro modelo seguindo a perspectiva original de três dimensões do instrumento e um segundo modelo, considerando uma natureza unidimensional do inventário. A avaliação dos modelos foi conduzida considerando os índices de ajuste $X^{2} /$ gl, Comparative Fit Index (CFI), Tucker Lewis Index (TLI) e Root Mean Square Error of Approximation (RMSEA). Os coeficientes são interpretados considerando os índices comparativos próximos de 1,00, enquanto $X^{2} / g l$, inferior a 5,00, e RMSEA inferior a 0,08 (Brown, 2015). Para evidências de precisão do instrumento recorreu-se ao indicadores dos coeficientes alfa de Cronbach e Ômega (Trizano-Hermosilla \& Alvarado, 2016).

Com o objetivo de oferecer informações sobre a invariância métrica da escala de fadiga, foi utilizada uma abordagem Multiple Indicators Multiple Causes (MIMIC), que consiste na inserção de uma variável de grupo como preditor da variável latente sendo testada. O segundo passo da avaliação por MIMIC consistiu ainda na inserção dos pesos de regressão especificamente para os itens, com o objetivo de verificar se a variável de grupo impacta especificamente neste nível. Foram observados como parâmetros (a) se a variável de grupo de fato oferece um preditor de funcionamento diferenciado da medida para o grupo de referência (no caso, ocupação militar) e (b) se os índices de ajuste do modelo apresentam deterioração indicando que a inserção do grupo como preditor insere em maior proporção erro ao invés de modelar a variância dos indicadores testados - neste sentido são testados ambos os escores produzidos pelas variáveis latentes do modelo no primeiro passo, e as cargas fatoriais da estrutura, no passo seguinte (Chun et al, 2016; Ferreira et al, 2016).

\section{Análise Fatorial Confirmatória}

Em análise preliminar o teste de Schwarz apontou para a retenção de três fatores em função do valor de $\mathrm{BIC}=905,03$, havendo para o modelo de dois fatores $\mathrm{BIC}=1011,15$ e para $\mathrm{O}$ modelo de quatro fatores $\mathrm{BIC}=993,01$. O modelo de um fator, concorrente ao original, apresentou uma degradação expressiva da informação para BIC $=1060,51$. Nesta direção foram testados dois modelos com o IFT-3D (original e concorrente).

O primeiro modelo (M1) ou Modelo original, apresentou três fatores de fadiga (física, cognitiva e emocional), seguindo a perspectiva do seu estudo de desenvolvimento (Frone \& Tidwell, 2016). Os resultados demonstraram indicadores de ajuste aceitáveis e adequados $\left(X^{2} / g l=3,95\right.$; CFI $=0,92$, TLI $=$ 0,91 e RMSEA=0,08 [0,07-0,09]). Por sua vez, o modelo (M2), foi estabelecido como estrutura concorrente e organizada a partir da estrutura de um único fator geral de fadiga. Os indicadores de (M2) foram menos ajustados e alguns índices inaceitáveis $\left(X^{2} /\right.$ $g l=9,47$; CFI $=0,78$; TLI $=0,75$ e RMSEA $=0,14[0,13-0,15])$. A tabela 2 apresenta os fatores finais, itens e respectivas cargas fatoriais dos itens.

Como observado na tabela 1 , os fatores resultantes obtiveram boas cargas fatoriais e índices de ajuste aceitáveis para versão de três dimensões do instrumento. $\mathrm{O}$ fator 1 , relacionado a fadiga física, contemplou os itens de avaliação de demandas de cansaço, exaustão e esgotamento físico associada as atividades do dia do trabalho, obtendo uma boa confiabilidade $(\alpha=0,92 ; \omega=0,91)$. $\mathrm{O}$ segundo fator correspondeu ao domínio de fadiga mental e obteve adequada precisão $(\alpha=0,94 ; \omega=0,94)$, sendo sua operacionalização representante de aspectos do esgotamento e exaustação mental nas tarefas do cotidiano laboral. Por fim, o terceiro fator contemplou a dimensão de fadiga emocional e foi composto por itens que avaliam a fadiga na esfera afetiva, envolvendo indicadores de cansaço, esgotamento e exaustão emocional $(\alpha=0,96 ; \omega=0,96)$.

\section{Invariância Métrica por Natureza da Ocupação}

Os dados foram analisados ainda considerando a invariância da medida por MIMIC, uma vez que o tamanho da amostra não permitiu uma análise multigrupo por ajuste dos modelos separadamente para cada um dos três grupos individualmente (segurança pública, saúde e população geral). Neste sentido foi observado que em um primeiro modelo, regredindo a natureza da ocupação enquanto militar x não militar sobre os fatores, efeitos significativos foram observados em todos os casos. Para o fator F1, foi observado um $\lambda=0,11$ ( $p=0,027)$, enquanto os demais fatores apresentaram coeficientes positivos mais elevados (F2, $\lambda=0,29$, $p<0,001 ; \mathrm{F} 3, \lambda=0,29, p<0,001)$. Isso significa que para os escores produzidos pelo instrumento nos três fatores, a amostra militar tende a apresentar uma superestimativa dos indicadores. Por outro lado, o modelo resulta em um ajuste que se deteriora para o RMSEA de 0,100 , indicando que a inserção da variável não otimiza o funcionamento da medida, o que seria um indicativo de funcionamento diferencial consistente para o grupo.

Outra informação relevante é que quando o indicador de grupo regride sobre os itens apenas, os coeficientes para os itens FAD1, FAD3 e FAD5, são os únicos que resultam não significativos, porém todos apresentam valores inferiores a 0,30 , exceto o item FAD10 $(\lambda=0,36, p<0,001)$. O mesmo fenômeno é observado, ocorrendo uma depreciação do RMSEA para um ajuste de 0,100 indicando que a inserção da variável grupo pode não estar modelando de forma suficiente a variância para o modelo. 


\begin{tabular}{|c|c|c|c|}
\hline Itens & F1 & $\mathrm{F} 2$ & F3 \\
\hline \multicolumn{4}{|l|}{ Fadiga física } \\
\hline $\begin{array}{l}\text { Teve dificuldade em participar de uma atividade } \\
\text { física no final do dia de trabalho? }\end{array}$ & 0,72 & & \\
\hline $\begin{array}{l}\text { Se sentiu fisicamente cansado no final do dia de } \\
\text { trabalho? }\end{array}$ & 0,70 & & \\
\hline $\begin{array}{l}\text { Se sentiu fisicamente esgotado no final do dia de } \\
\text { trabalho? }\end{array}$ & 0,86 & & \\
\hline $\begin{array}{l}\text { Quis fisicamente desmoronar no final do dia de } \\
\text { trabalho? }\end{array}$ & 0,84 & & \\
\hline $\begin{array}{l}\text { Se sentiu fisicamente exausto no final do dia de } \\
\text { trabalho? }\end{array}$ & 0,90 & & \\
\hline $\begin{array}{l}\text { Quis evitar qualquer situação que exigisse o uso de } \\
\text { muita energia física no final do dia de trabalho? }\end{array}$ & 0,78 & & \\
\hline \multicolumn{4}{|l|}{ Fadiga mental } \\
\hline $\begin{array}{l}\text { Se sentiu mentalmente cansado no final do dia de } \\
\text { trabalho? }\end{array}$ & & 0,82 & \\
\hline $\begin{array}{l}\text { Teve dificuldade de pensar ou de se concentrar no } \\
\text { final do dia de trabalho? }\end{array}$ & & 0,84 & \\
\hline $\begin{array}{l}\text { Se sentiu mentalmente esgotado no final do dia de } \\
\text { trabalho? }\end{array}$ & & 0,91 & \\
\hline $\begin{array}{l}\text { Quis mentalmente desmoronar no final do dia de } \\
\text { trabalho? }\end{array}$ & & 0,84 & \\
\hline $\begin{array}{l}\text { Se sentiu mentalmente exausto no final do dia de } \\
\text { trabalho? }\end{array}$ & & 0,90 & \\
\hline $\begin{array}{l}\text { Quis evitar qualquer situação que exigisse o uso de } \\
\text { muita energia mental no final do dia de trabalho? }\end{array}$ & & 0,85 & \\
\hline \multicolumn{4}{|l|}{ Fadiga emocional } \\
\hline $\begin{array}{l}\text { Se sentiu emocionalmente cansado no final do dia } \\
\text { de trabalho? }\end{array}$ & & & 0,89 \\
\hline $\begin{array}{l}\text { Teve dificuldade em demonstrar e lidar com emo- } \\
\text { ções no final do dia de trabalho? }\end{array}$ & & & 0,87 \\
\hline $\begin{array}{l}\text { Se sentiu emocionalmente esgotado no final do dia } \\
\text { de trabalho? }\end{array}$ & & & 0,96 \\
\hline $\begin{array}{l}\text { Quis emocionalmente desmoronar no final do dia } \\
\text { de trabalho? }\end{array}$ & & & 0,89 \\
\hline $\begin{array}{l}\text { Se sentiu emocionalmente exausto no final do dia } \\
\text { de trabalho? }\end{array}$ & & & 0,92 \\
\hline $\begin{array}{l}\text { Quis evitar qualquer situação que exigisse o uso } \\
\text { de muita energia emocional no final do dia de } \\
\text { trabalho? }\end{array}$ & & & 0,90 \\
\hline Coeficiente de precisão alfa de Cronbach & 0,92 & 0,94 & 0,96 \\
\hline Coeficiente de precisão Ômega & 0,91 & 0,94 & 0,96 \\
\hline
\end{tabular}

\section{Evidências Externas de Validade do 3D-WFI}

Concluindo os achados iniciais de validade da Three-Dimensional Work Fatigue Inventory (3D-WFI), procurou-se avaliar sua relação com variáveis externas. Desta foram executadas correlações do tipo $r$ de Pearson entre as três dimensões de fadiga no trabalho (física, cognitiva e emocional) e os construtos: conflito trabalhofamília (trabalho interferindo na família e; família interferindo no trabalho), engajamento no trabalho e satisfação com a vida.

Com base na tabela 3 observam-se correlações elevadas entre todas as dimensões de fadiga $(r>0,70 ; p<0,001)$ da 3D-WFI. Especificamente constatou-se que a dimensão fadiga física obteve correlação negativa com trabalho interferindo na família $(r=$ $0,45 ; p<0,001)$ e positiva com satisfação com a vida $(\mathrm{r}=0,31 ; p$ $<0,001)$ e engajamento no trabalho $(r=0,34 ; p<0,001)$. Fadiga cognitiva, por sua vez, correlacionou-se de maneira negativa com trabalho interferindo na família $(r=-0,38 ; p<0,001)$ e de modo positivo com satisfação com a vida $(r=0,17, p<0,001)$ e engaja-
Tabela 3

Dados descritivos e correlacionais da 3D-WFI.

\begin{tabular}{lrrrrrrrrr}
\hline \multicolumn{1}{c}{ Variáveis } & $M$ & $D P$ & 1 & 2 & 3 & 4 & 5 & 6 & 7 \\
\hline 1. F. Fís. & 2,68 & 1,08 & 1 & & & & & & \\
2. F. Cogn. & 2,77 & 1,15 & $0,71^{* *}$ & 1 & & & & & \\
3. F. Emoc. & 3,14 & 1,26 & $0,70^{* *}$ & $0,81^{* *}$ & 1 & & & & \\
4. TIF & 2,83 & 1,25 & $-0,45^{* *}$ & $-0,38^{* *}$ & $-0,35^{* *}$ & 1 & & & \\
5. FIT & 1,82 & 0,97 & $-0,04$ & $-0,09$ & $-0,10$ & $0,30^{* *}$ & 1 & & \\
6. Sat. vida & 3,75 & 1,01 & $0,31^{* *}$ & $0,17^{* *}$ & $0,25^{* *}$ & - & - & 1 & \\
7. Eng. & 3,47 & 0,83 & $0,34^{* *}$ & $0,27^{* *}$ & $0,27^{* *}$ & $-0,23^{* *}$ & $-0,21^{* *}$ & - & 1 \\
Trab. & & & & & & & & & \\
\hline
\end{tabular}

Nota. Legenda: F. Fís. (fadiga física); F. Cogn. (fadiga cognitva); F. Emoc. (fadiga emocional); TIF (trabalho interferindo na família); FIT (família interferindo no trabalho); Sat. Vida (satisfação com a vida) e; Eng. Trab. (engajamento no trabalho). ${ }^{* *} p<0,001$.

mento no trabalho $(r=0,27 ; p<0,001)$. Finalmente, a dimensão de fadiga emocional, associou-se de maneira inversa com trabalho interferindo na família $(r=-0,35 ; p<0,001)$ e de maneira direta com satisfação com a vida $(r=0,25 ; p<0,001)$ e engajamento no trabalho $(r=0,23 ; p<0,001)$. Não foram observadas relações significativas entre as dimensões da $3 D-W F I$ e de família interferindo no trabalho $(p>0,05)$.

\section{Discussão}

O presente artigo teve como objetivo principal adaptar e levantar as propriedades psicométricas de validade (estrutura interna e externa) e precisão para o contexto brasileiro da ThreeDimensional Work Fatigue Inventory (3D-WFI). No processo de condução da pesquisa, foram explorados e confirmados aspectos da estrutura interna do instrumento no contexto brasileiro, testada a invariância segundo natureza ocupacional, obtidos indicadores de precisão e levantadas evidências externas com construtos teóricos pertinentes (conflito trabalho-família, satisfação com vida e engajamento no trabalho), sendo verificada congruência com a perspectiva original de três dimensões de fadiga no trabalho (física, cognitiva e emocional) (Frone \& Tidwell, 2016), além da obtenção de evidências semelhantes aos estudos realizados na Alemanha (Frone et al., 2018), Líbia (Obeid et al., 2019) e Noruega (Knutsen, 2019).

O inventário de fadiga no trabalho, na sua versão português do Brasil, evidenciou bons índices de ajuste nos parâmetros avaliados na análise fatorial confirmatória ( $X^{2} / g l$, CFI, TLI, RMSEA) (Brown, 2015), para estrutura interna de três dimensões de fadiga (física, cognitiva e emocional). Ao contrastar a qualidade métrica do instrumento adaptado ao contexto brasileiro com a versão americana (Frone \& Tidwell, 2016) e alemã (Frone et al., 2018), percebe-se que os elementos técnicos do instrumento repercutem resultados similares de ajuste da $3 D$-WFI, assim como entre as outras versões culturais do instrumento (Frone et al., 2018; Obeid et al., 2019; Knutsen, 2019). Essas informações que corroboram a hipótese 1 do estudo.

Do ponto de vista teórico e de evidências de validade externa, verifica-se que todas as dimensões de fadiga se relacionaram de maneira elevada entre si, indicando que a fadiga é um elemento de cansaço e perda de energia que conserva aspectos comuns e extensivo entre domínios cognitivos, emocionais e físico (Frone et al., 2018). As dimensões de fadiga obtiveram também correlação inversa com aspectos de trabalho interferindo na família do construto conflito trabalho-família (Aguiar \& Bastos, 2013), não sendo observada relação significativa com família interferindo no trabalho, corroborando parcialmente a hipótese 2. Nesta direção, percebe-se que quanto maior a percepção de fadiga dos indivíduos, maior incidência de tensionamentos da dimensão de trabalho 
interferir nos aspectos das vivências da família. A relação entre aumento de demandas percebidas no trabalho e consequências para domínios externos não trabalho, como família, por exemplo, foi relatada por Akinbode et al (2018), o qual apontou a interdependência entre os domínios. Netemeyer, Boles e Mc Murrian (1996), apontam que indicadores de esgotamento laboral, como, por exemplo, tensão e desagaste no trabalho foram associados com conflito trabalho-família no estudo com norte-americanos.

Por sua vez, a ausência de correlações entre o domínio de interferência da família no trabalho e dimensões de fadiga não atendem plenamente achados anteriores que apontam que aspectos do ambiente de trabalho afetam mutuamente domínios de trabalho e família (De Andrade et al., 2020; De Andrade, Ziebell de Oliveira, $\&$ Hatfiel, 2017). Sugerem-se futuros estudos que possam explorar melhor estes mecanismos com fadiga no trabalho, como, por exemplo, o teste de dimensões moderadoras da atividade ocupacional e esta condição individual de desgaste no trabalho.

A relação evidenciada entre as dimensões de fadiga no trabalho e satisfação com a vida corroboram a hipótese 3 do estudo. $\mathrm{O}$ estado psicológico de fadiga no trabalho indica a percepção pessoal de cansaço, exaustação e diminuição de aspectos de energia do indivíduo (Frone \& Tidwell, 2016). Num contexto temporal de médio prazo de exposição a elementos de tensão no trabalho é esperado o transbordamento de percepções negativas para outros contextos da vida além do trabalho (ex. família). Conforme Grawitch et al. (2013) existe uma rede nomológica de aspectos de equilíbrio trabalho e vida, na qual se identificam a interdependência e os efeitos entre os distintos domínios. A relação observada entre dimensões de fadiga e satisfação a vida no trabalho na literatura da área (Erdogan, Bauer, Truxillo, \& Mansfield, 2012) e nos resultados do presente estudo são uma evidência teórico e prática para o diagnóstico e intervenção de profissionais, pesquisadores e gestores organizacionais sobre o tema.

Por sua vez, a relação encontrada entre o construto engajamento com o trabalho e as dimensões de fadiga no trabalho corroboram a hipótese relacional quatro do estudo. Tais resultados indicam que o estado de absorção e vigor positivo característico do engajamento torna-se enfraquecidos com o aumento dos aspectos de fadiga. Resultados semelhantes foram apontados no estudo com trabalhadores alemães (Frone et al., 2018), no qual as dimensões de fadiga emocional, física e cognitiva foram preditores de menor engajamento no trabalho. Profissionais fadigados são ainda caracterizados por possuírem menor energia, menos prazer e busca por desafios, além da menor percepção de habilidades e identificação com o trabalho (Salanova, Del Líbano, Llorens, \& Schaufeli, 2014).

A Three-Dimensional Work Fatigue Inventory (3D-WFI), adaptada ao contexto brasileiro neste estudo, soma-se ao hall de outras ferramentas de avaliação de fadiga no trabalho disponíveis para avaliação do construto na população brasileira (Cho et al., 2007; Gouveia et al., 2015; Moriguchi et al., 2012; Mota et al., 2009). Importante mencionar que, além de atual, a 3D-WFI apresenta, entre outras vantagens, ao ser comparada como outras ferramentas disponíveis, a proposta de ser um instrumento generalista de avaliação para fadiga, ou seja, permite o uso em diferentes contextos ocupacionais. Aspecto corroborado com os testes de invariância da medida em três grupos amostrais diferentes (segurança pública, hospitais e geral), bem como sua natureza semântica de itens adaptados ao cenário do português brasileiro.

Para futuros estudos, indicam-se a realização de estudos preditivos com outras populações ocupacionais, sendo essa uma limitação do presente trabalho, o qual, mesmo incluindo na amostra profissionais de contextos de risco e frequente tensão (segurança pública e saúde), não atentou a uma análise de outras categorias profissionais (ex. profissionais de construção civil, professores, entre outros). Sugere-se também o incremento de evidências de validade com construtos do campo de saúde laboral, como estresse, ansiedade, depressão e burnout (Cortez, Zerbini, \& Magali, 2019), bem como fenômenos adicionais do campo da psicologia positiva organizacional (Vazquez et al., 2019), como, por exemplo, capital psicológico e interpessoal, como civilidade organizacional(De Andrade, Matos, Lobianco, \& Broseguini, 2020)

Por fim, conclui-se esse trabalho recomendando o uso da Three-Dimensional Work Fatigue Inventory (3D-WFI) para pesquisa e diagnóstico no campo de saúde e trabalho. O construto fadiga e sua medida apresentados neste estudo favorecem o entendimento das consequências e extensões de fenômenos como estresse (Frone et al., 2018), burnout, incivilidade no trabalho (De Andrade et al., 2020), bem como pode contribuir para o entendimento de questões que extrapolam as dimensões do trabalho e ocasionam efeitos em domínios da vida pessoa, conflito trabalho-família (De Andrade, Moraes, et al., 2020).

\section{Referências}

Aguiar, C., \& Bastos, V. B. (2013). Tradução, adaptação e evidências de validade para a medida de Conflito trabalho-família. Avaliação Psicológica, 12(2), 203-212. Recuperado de http://www.redalyc.org/pdf/3350/335027505011. pdf

Akinbode, G. A., Folusho, A., \& Isrsel, U. N. (2018). Psychopathology of Work-Family and Family-Work Conflicts among Dual Income Earners : An Empirical Analysis from Cosmopolitan Lagos. Advances in Social Sciences Research Journal, 5(3), 289-314. https://doi.org/10.14738/assri.53.4193

Bauerle, T., Dugdale, Z., \& Poplin, G. (2018). Mineworker fatigue: A review of what we know and future directions. Mining Engineering, 2018-Febru(3), 1-19.

Borsa, J. C., Damásio, B. F., \& Bandeira, D. R. (2012). Adaptação e validação de instrumentos psicológicos entre culturas: Algumas considerações. Paideia, 22(53), 423-432. https://doi.org/10.1590/1982-43272253201314

Celestino, V. R. R., Bucher-Maluschke, J. S. N. F., \& Marqueze, E. C. (2020). Fatigue at work: Scale validation with airline pilots. $B A R$ - Brazilian Administration Review, 17(3), 1-24. https://doi.org/10.1590/18077692 bar2020190031

Chen, M. L., Lu, S. Y., \& Mao, I. F. (2019). Subjective symptoms and physiological measures of fatigue in air traffic controllers. International Journal of Industrial Ergonomics, 70(110), 1-8. https://doi.org/10.1016/j.ergon.2018.12.004

Cho, H. J., Costa, E., Menezes, P. R., Chalder, T., Bhugra, D., \& Wessely, S. (2007). Cross-cultural validation of the Chalder Fatigue Questionnaire in Brazilian primary care. Journal of Psychosomatic Research, 62(3), 301-304. https://doi. org/10.1016/i.jpsychores.2006.10.018

Cortez, P. A., Zerbini, T., \& Magali, H. (2019). Work Context and Burnout : Confirmation of Moderators from Meta-analysis Evidence. Revista Psicologia: Organizações e Trabalho, 19, 755-761. https://doi.org/10.17652/ rpot/2019.4.17499

De Andrade, A. L., Matos, F. R., Lobianco, V. M. P., \& Broseguini, G. B. (2020). (In) civilidade no Trabalho: Medidas e Modelos. Revista Psicologia: Organizações e Trabalho, 20(1), 1-8. https://doi.org/10.17652/rpot/2020.1.16841

De Andrade, A. L., Moraes, T. D., Martins-Silva, P. O., \& Queiroz, S. S. de. (2020). Conflito trabalho-família em profissionais do contexto hospitalar: análise de preditores. Revista de Psicología, 38(2), 451-478. https://doi.org/https://doi. org $/ 10.18800 /$ psico. 202002.004

De Andrade, A. L., Ziebell de Oliveira, M., \& Hatfield, E. (2017). Conflito trabalho-família: Um estudo com brasileiros e norte-americanos. Revista Psicologia: Organizações e Trabalho, 17(2), 106-113. https://doi.org/10.17652/ rpot/2017.2.12738

De Vries, J., Michielsen, H., Van Heck, G. L., \& Drent, M. (2004). Measuring fatigue in sarcoidosis: The Fatigue Assessment Scale (FAS). British Journal of Health Psychology, 9(3), 279-291. https://doi. org/10.1348/1359107041557048

Diener, E., Emmons, R. A., Larsem, R. J., \& Griffin, S. (1985). The Satisfaction With Life Scale. Journal of Personality Assessment, 49(1), 71-75. https://doi. org/10.1207/s15327752jpa4901 13

Erdogan, B., Bauer, T. N., Truxillo, D. M., \& Mansfield, L. R. (2012). Whistle While You Work: A Review of the Life Satisfaction Literature. Journal of Management (Vol. 38, pp. 1038-1083). https://doi.org/10.1177/0149206311429379

Fan, J., \& Smith, A. P. (2019). Mental Workload and Other Causes of Different Types of Fatigue in Rail Staff. Em Communications in Computer and Information Science (Vol. 1012). https://doi.org/10.1007/978-3-030-14273-5 9 
Fourie, R., \& Keyser, E. (2018). The Relationship Between Blue Collar Employee Fatigue. International Journal Of Social Sciences And Humanity Studies, 10(1), 205-222.

Frone, M. R., \& Blais, A.-R. (2020a). Organizational Downsizing, Work Conditions, and Employee Outcomes: Identifying Targets for Workplace Intervention among Survivors. International Journal of Environmental Research and Public Health, 17(3), 719. https://doi.org/10.3390/ijerph17030719

Frone, M. R., \& Blais, A. R. (2020b). Organizational downsizing, work conditions, and employee outcomes: Identifying targets for workplace intervention among survivors. International Journal of Environmental Research and Public Health, 17(3). https://doi.org/10.3390/ijerph17030719

Frone, M. R., Reis, D., \& Ottenstein, C. (2018). A German version of the ThreeDimensional Work Fatigue Inventory (3D-WFI): Factor structure, internal consistency, and correlates. Stress and Health, 34(5), 674-680. https://doi. org $/ 10.1002 / \mathrm{smi} .2828$

Frone, M. R., \& Tidwell, M.-C. O. . (2016). The Meaning and Measurement of Work Fatigue: Development and Evaluation of the Three-Dimensional Work Fatigue Inventory (3D-WFI). Journal of Occupational Health Psychology, 20(3), 273-288. https://doi.org/10.1037/a0038700.The

Gouveia, V. V., Oliveira, G. F. de, Mendes, L. A. de C., Souza, L. E. C. de, Cavalcanti, T. M., \& Melo, R. L. P. de. (2015). Escala de avaliação da fadiga: adaptação para profissionais da saúde. Revista Psicologia Organizações e Trabalho, 15(3), 246-256. https://doi.org/10.17652/rpot/2015.3.594

Grawitch, M. J., Maloney, P. W., Barber, L. K., \& Mooshegian, S. E. (2013). Examining the nomological network of satisfaction with work-Life balance. Journal of Occupational Health Psychology, 18(3), 276-284. https://doi. org/10.1037/a0032754

Hobfoll, S. E. (1989). Conservation of resources: A new attempt at conceptualizing stress. American Psychologist, 44(3), 513-524. https://doi. org/10.1037/0003-066X.44.3.513

Husain, N. A., Mohamad, J., \& Idris, M. A. (2019). Daily emotional demands on traffic crashes among taxi drivers: Fatigue and safety motivation as mediators. LATSS Research, 43(4), 268-276. https://doi.org/10.1016/i. iatssr.2019.03.001

International Test Comission. (2017). The ITC Guidelines for Translating and Adapting Tests. Version $2.4\left(2^{a}\right.$ ed.). 1-41. Recuperado de https://www.intestcom.org/ files/guideline test adaptation 2ed.pdf

Knutsen, R. H. (2019). The Relationship between Organizational Climate, Perceived Organizational Support, Employee Participation, and Readiness for Change within the Norwegian Police Service (Dissertação de Mestrado). Universidade de Oslo, Noruega.

Moriguchi, C. S., Trevizani, T., de Fátima Carreira Moreira, R., Januário, L. B., de Oliveira, A. B., \& Coury, H. J. C. G. (2012). Need for recovery assessment among nursing professionals and call center operators. Work, 41(SUPPL.1), 4838-4842. https://doi.org/10.3233/WOR-2012-0773-4838

Mota, D. D. C. F., Pimenta, C. A. M., \& Piper, B. F. (2009). Fatigue in Brazilian cancer patients, caregivers, and nursing students: a psychometric validation study of the Piper Fatigue Scale-Revised. Supportive Care in Cancer, 17(6), 645-652. https://doi.org/10.1007/s00520-008-0518-x

Netemeyer, R. G., Boles, J. S., \& McMurrian, R. (1996). Development and validation of work-family conflict and family-work conflict scales. Journal of Applied Psychology, 81(4), 400-410. https://doi.org/10.1037/0021$\underline{9010.81 .4 .400}$

Obeid, S., Akel, M., Haddad, C., Fares, K., Sacre, H., Salameh, P., \& Hallit, S. (2019a). Factors associated with alexithymia among the Lebanese population: Results of a cross-sectional study. BMC Psychology, 7(1). https:// doi.org/10.1186/s40359-019-0353-5

Obeid, S., Akel, M., Haddad, C., Fares, K., Sacre, H., Salameh, P., \& Hallit, S. (2019b). Factors associated with alexithymia among the Lebanese population: Results of a cross-sectional study. BMC Psychology, 7(1), 1-10. https://doi.org/10.1186/s40359-019-0353-5

Patterson, P. D., Weaver, M. D., Fabio, A., Teasley, E. M., Renn, M. L., Curtis, B. R., ... Higgins, J. S. (2018). Reliability and Validity of Survey Instruments to Measure Work-Related Fatigue in the Emergency Medical Services Setting: A Systematic Review. Prehospital Emergency Care, 22, 17-27. https://doi.org/1 $\underline{0.1080 / 10903127.2017 .1376134}$

Powell, G. N., \& Greenhaus, J. H. (2010). Sex, Gender, and the Work-toFamily Interface: Exploring Negative and Positive Interdependencies. Academy of Management Journal, 53(3), 513-534. https://doi.org/10.5465/ AMJ.2010.51468647

Salanova, M., Del Líbano, M., Llorens, S., \& Schaufeli, W. B. (2014). Engaged, workaholic, burned-out or just 9-to-5? Toward a typology of employee wellbeing. Stress and Health, 30(1), 71-81. https://doi.org/10.1002/smi.2499

Stuetzle, K. V., Pavlin, B. I., Smith, N. A., \& Weston, K. M. (2018). Survey of Occupational Fatigue in Anaesthetists in Australia and New Zealand. Anaesthesia and Intensive Care, 46(4), 414-423. https://doi. org/10.1177/0310057X1804600411
Trizano-Hermosilla, I., \& Alvarado, J. M. (2016). Best alternatives to Cronbach's alpha reliability in realistic conditions: Congeneric and asymmetrical measurements. Frontiers in Psychology, 7(MAY), 1-8. https://doi.org/10.3389/ fpsyg.2016.00769

Vazquez, A. C. S., Ferreira, M. C., \& Mendonça, H. (2019). Avanços na Psicologia Positiva: Bem-Estar, Engajamento e Redesenho no Trabalho. Avaliação Psicológica, 18(51), 343-351.

Westman, M., Hobfoll, S. E., Chen, S., Davidson, O. B., \& Laski, S. (2015). Organizational stress through the lens of Conservation of Resources (CoR) Theory. Em Exploring Interpersonal Dynamics (pp. 167-220). https://doi. org/10.1016/S1479-3555(04)04005-3

Zanon, C., Bardagi, M. P., Layous, K., \& Hutz, C. S. (2014). Validation of the Satisfaction with Life Scale to Brazilians: Evidences of Measurement Noninvariance Across Brazil and US. Social Indicators Research, 119(1), 443-453. https://doi.org/10.1007/s11205-013-0478-5

\section{Informações sobre os autores:}

Apoio. Bolsa de Produtividade CNPq para o primeiro e segundo autor. Suporte para condução da pesquisa pelo Edital Universal e Taxa de Pesquisa da Fundação de Amparo à Pesquisa e Inovação do Espírito Santo (FAPES).

\section{Alexsandro Luiz De Andrade}

CEMUNI VI Universidade Federal do Espírito Santo - UFES

Av. Fernando Ferrari, 514, Goiabeiras

29075-910 Vitória, ES, Brasil

E-mail: alexsandro.deandrade@yahoo.com

\section{Marcos Aguiar de Souza}

E-mail: maguiarsouza@uol.com.br

\section{Pedro Paulo Pires}

E-mail: ppires85@gmail.com 